\title{
A Basic ANN System for Prediction of Excess Air Coefficient on Coal Burners Equipped with a CCD Camera
}

\author{
Cem Onat ${ }^{1, *}$, Mahmut Daskin ${ }^{2}$ \\ ${ }^{1}$ Department of Airframe and Power Plant, Firat University, Turkey \\ ${ }^{2}$ Department of Mechanical Engineering, Inonu University, Turkey
}

Copyright $@ 2019$ by authors, all rights reserved. Authors agree that this article remains permanently open access under the terms of the Creative Commons Attribution License 4.0 International License

\begin{abstract}
Excess air coefficient $(\lambda)$ is the most important parameter characterizing the combustion efficiency. Conventional measurement of $\lambda$ is practiced by way of the flue analyze device with high market priced. Estimating of the $\lambda$ from flame images is crucial in perspective of the combustion control because of decreasing structural dead time of the combustion process. Beside, estimation systems can be used continuously in a closed loop control system, unlike conventional analyzers. This paper represents a basic $\lambda$ prediction system with a neural network for small scale nut coal burner equipped with a CCD camera. The proposed estimation system has two inputs. First input is stack gas temperature simply measuring from the flue. To choose the second input, eleven different matrix parameters have been evaluated together with flue gas temperature values and performed by matrix-based multiple linear regression analysis. As a result of these analyses, it has been seen that the trace of image matrix obtained from the flame image provides higher accuracy than the other matrix parameters. This instantaneous trace value of image source matrix is then filtered from high frequency dynamics by means of a low pass filter. Experimental data of the inputs and $\lambda$ are synchronously matched by a neural network. Trained algorithm has reached $\mathrm{R}=0.984$ in terms of accuracy. It is seen from the result that proposed estimating system using flame image with assistance of the stack gas temperature can be preferred in combustion control systems.
\end{abstract}

Keywords Combustion, Excess Air Coefficient, Image Processing, Neural Network, Multi Linear Regression, Combustion Control

\section{Introduction}

Burner systems have been widely used in industries and domestic areas for steam production and hot water supply [1-3]. In recent years, engineers have a motivation for improving combustion efficiency due to higher fuel costs and occasionally limited fuel availability. The combustion process is extremely complicated, because combustion condition is very changeable [4]. When it changes, the excess air will deviate from the optimal value; correspondingly, the combustion system will deviate from the optimal state. Therefore, the effectiveness of combustion control has become more and more important.

Excess air coefficient $(\lambda)$ is the most important parameter featuring the effectiveness of combustion. On the other hand, it represents an ideal value for maximization of combustion effectiveness. This is very precious for combustion control. $\lambda$ is conventionally measured by means of flue gas analyze devices. The measurement is based on a chemical process. The devices have high market price and operating cost. Also, they are not compatible for long term usage. On the contrary, the flame images can immediately reflect the current status of the combustors and so they are crucial in perspective of the combustion control because of decreasing structural dead time of the combustion process inasmuch as the dead time affects adversely disturbance rejection performance of closed loop control systems [5-8]. Due to the ability of the flame images, there is abundance of theory on flame image processing technology. Their approach is to compute radius and geometrical properties of the flame extracted from images, such as classifying the flame into arbitrarily defined states [9], estimating various quantities by flicker rate [10], $\mathrm{CO}_{2}$ and $\mathrm{NO}_{\mathrm{X}}$ emissions [11] and un-burnt carbon [12]. The studies are based on gray scale flame images. A few research works have analyzed the flames using bi-color method [13] and spectrometers [14] from which it is possible to extract more precise chemical information about the radicals present in the flame. A limitation of these approaches is related to extracting visual flame features 
directly from the image space.

In studies of flame image processing, radiant energy signal (RES) is used frequently [15-22]. RES is one of the important exhaustive parameters. It is simply obtained from a flame image, which can reflect the rapid variation in coal amount, and can also sense the transient change of coal quality. RES can be obtained by means of different approaches. For example, RES is an average gray value in the flame image in [15-17]. RES can also be a light intensity signal of the combustion chamber [18]. Researchers explained the flame temperature distribution and the RES are sensitive to change in the combustion of the boiler [19]. Also RES and combustion rate can be associated [20]. The relationship between fuel consumption rate and heat absorption rate can be achieved with RES [21]. However, RES is a considerable noisy with high frequency dynamics. This situation is unfavorable for combustion control. Besides, Talu et al. have shown that RES is not as good as competence for prediction of $\lambda$ [22]. The prediction algorithm with neural network proposed by Talu et al. has generated very noisy signal of $\lambda$ and have high computation load. In another recent study, PCA (Principle Component Analyze) and RWN (Random Weight Network) techniques have been used together [23]. Under different conditions, combustion state definition have been realized by the calculation based on gray level co-occurrence matrix and achieved 91\% accuracy. However, the method is quite complex and the accuracy is low. This is an important handicap in practice. Within this scope, this paper represents a basic and effective estimation system. Firstly, it is analyzed the correlation between air excess coefficient $(\lambda)$ and the filtered characteristic parameters of flame image captured from the nut coal burning chamber by means of a CCD camera. The regression analyses have performed on eleven different flame characteristic features by means of the matrix approach. These parameters have evaluated together with flue temperature. The derived characteristic features are the one norm, second norm, infinity norm, Frobenius norm, normalized sum of pixel values, matrix trace, matrix rank, sum of pixel values exceeding 30. Smallest eigen value, sum of the smallest 10 eigen values and sum of all eigen values of source matrix (the image). It has been found that the matrix trace has the most valuable correlation to the air excess coefficient. Finally, the temperature value $\left(T_{\text {flue }}\right)$ simply measuring from the flue and trace value of image source matrix which is filtered from high frequency dynamics by means of a low pass filter are simultaneously used in an artificial neural network (ANN). Trained algorithm has estimated $\lambda$ with $R=0.984$ in terms of accuracy. The proposed estimation system can be easily expanded to pulverized coal-fired large scale systems. Because the images of pulverized coal flame have a more homogeneous than the images of nut coal flame. In addition to this, the proposed system has a great potential for usage in closed loop combustion control systems due to its basic structure, high accuracy and producing the noiseless data.

The rest of the paper is organized as follows: In Section 1 , it is presented the experimental system. Experimental setup is given in Section 2. In section 3, it is given regression analyze between flame image properties assisted with the $\mathrm{T}_{\text {flue }}$ and $\lambda$. Section 4 describes the proposed data processing schema. Finally, section 5 concludes the paper.

\section{Experimental Setup}

The schematic representation of the experimental system is given at Figure 1. The system has consisted of eleven units. The spiral forwarder transports the nut coal in the bunker to combustion chamber. The spiral is driven by a mono-phase AC motor, and the fan is driven by a three phase AC motor. The fan supplies fresh air to the combustion chamber.

The images were captured from monitoring window by means of a CCD camera (Aptina MT9P031 with CMOS sensors Basler acA2500-14gc Gig E) mounted on a small sized automatic loading nut- coal burner system and $\lambda$ was measured by a gas analysis device (Testo), and also the flue temperature $\left(\mathrm{T}_{\text {flue }}\right)$ was synchronously measured every seconds. Thermal capacity of the burner is $85000 \mathrm{kcal} / \mathrm{h}$. The monitoring window of the combustion chamber is circular form with $12 \mathrm{~cm}$ diameter and the CCD camera frame rate was $14 \mathrm{fps}$. An $8 \mathrm{~mm}$ lens with a horizontal view angle of $78^{\circ}$ and a vertical view angle of $90^{\circ}$ is attached to the body of the camera. Localization of the CCD camera is illustrated by Figure 2 .

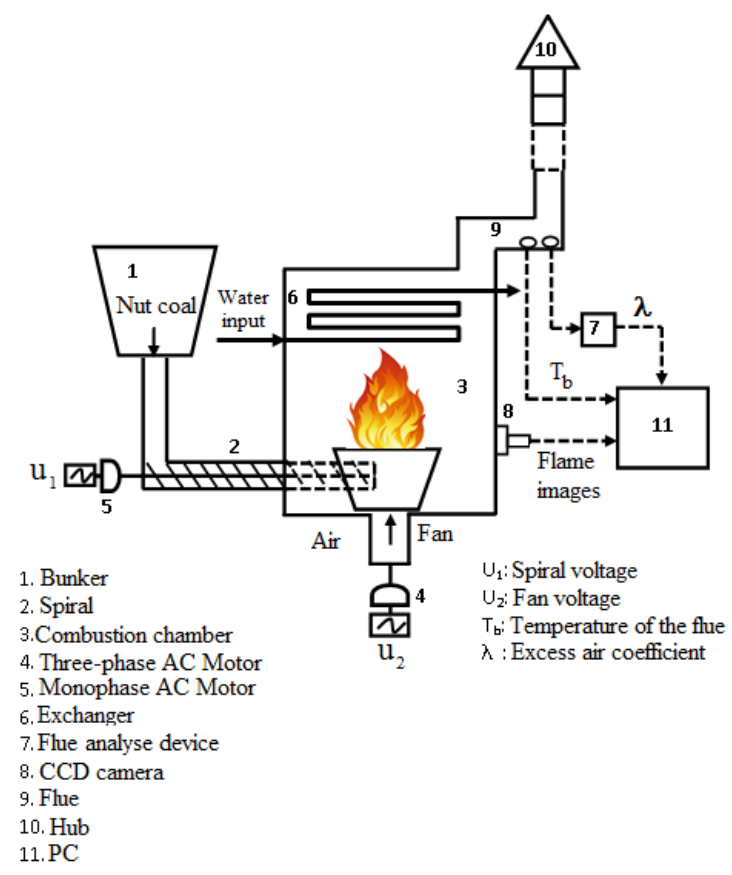

Figure 1. Schematic representation of the experimental system 


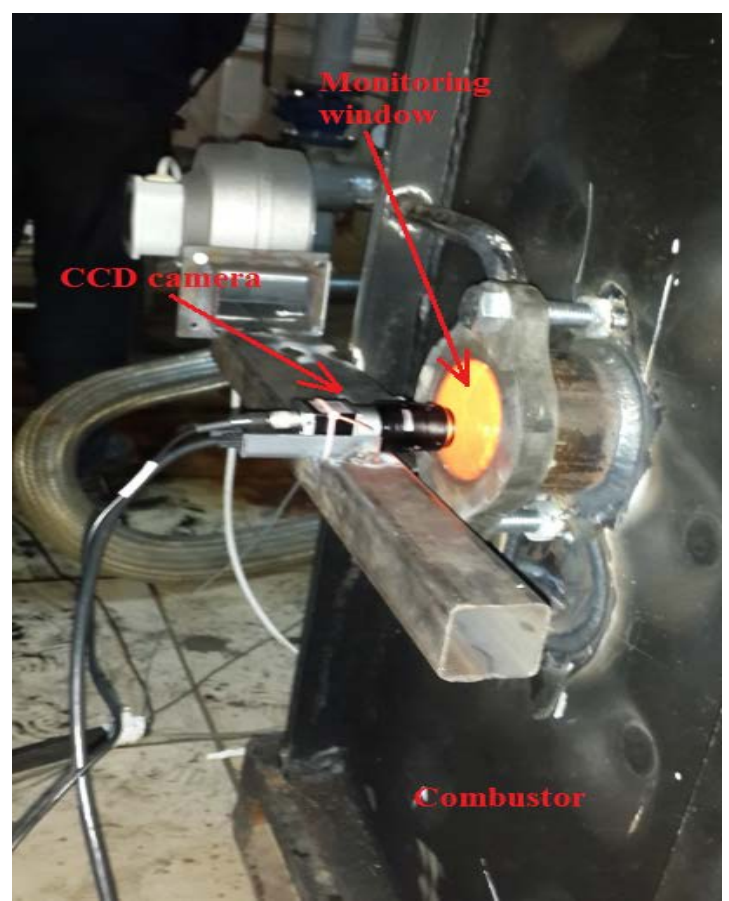

Figure 2. Localization of the CCD camera in the system

\section{Regression Analysis}

The features extracted out from the flame images obtained by the CCD camera were subjected to the regression analysis together with the information of the flue temperature after filtering by the low-pass filter given in Eq. (1).

$$
F(s)=\frac{1}{28 s^{2}+10 s+1}
$$

The one norm, two norm, infinity norm, frobenius norm, sum of normalized pixels, trace of matrix, rank of matrix, sum of the pixels with a threshold value greater than 30 , the smallest eigen value, sum of the smallest 10 eigen values and sum of all eigen values features extracted out from the images obtained from the CCD camera is given in Figure 3. These data taken from the camera are named $C_{1}, C_{2}, \ldots, C_{11}$ respectively. In order to eliminate the high frequency noises in the data, a low pass filter $F(s)$ which is given in Eq.1. is applied. Using the signal with high frequency dynamics in closed loop systems is challenge with regard to controller design. After filtering process this data normalized in the range $\left(\begin{array}{ll}0 & 1\end{array}\right)$. The filtered data are named $C_{1 f}, C_{2 f}, \ldots, C_{11 f}$ respectively, and are shown in Figure 4.
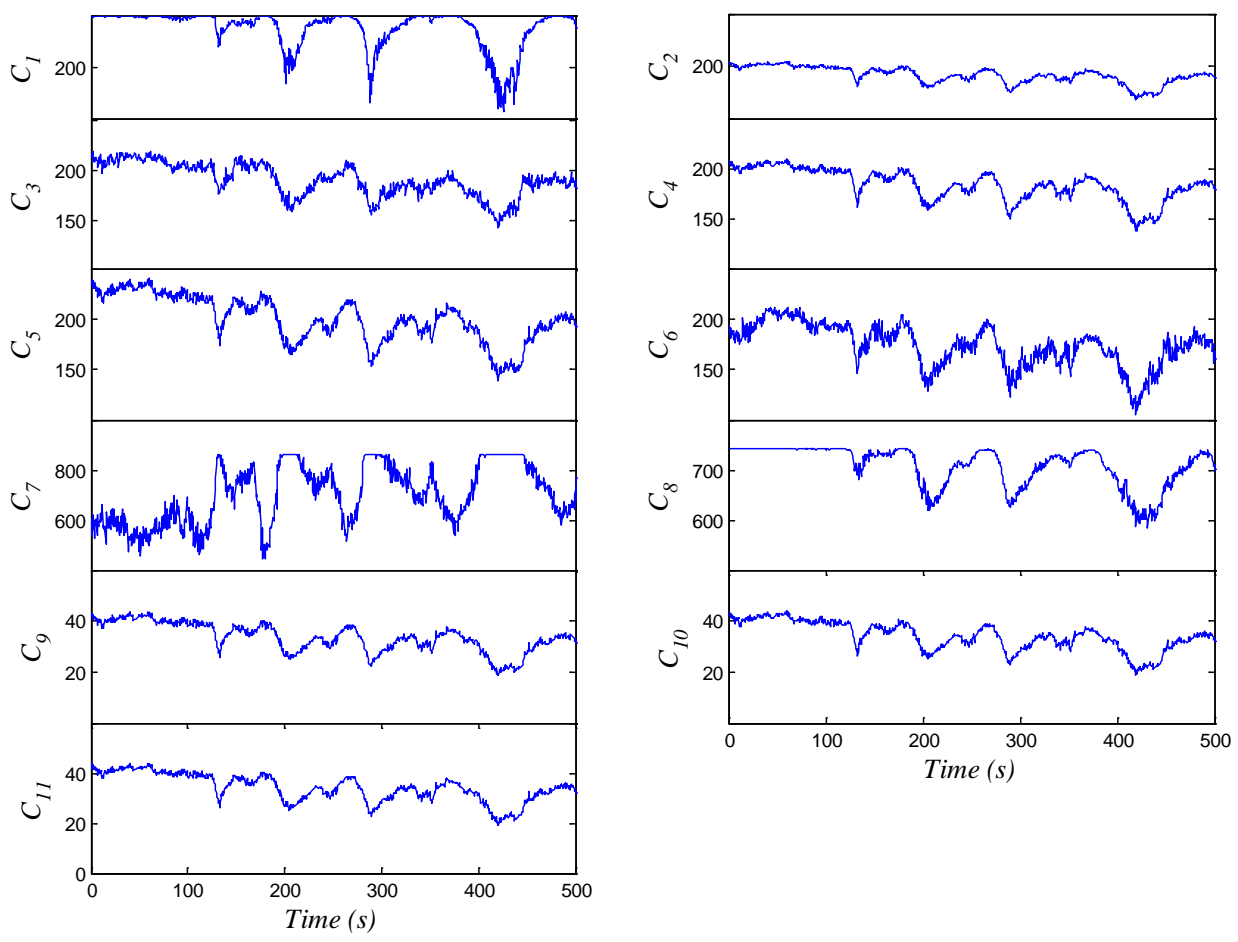

Figure 3. Raw flame image information 

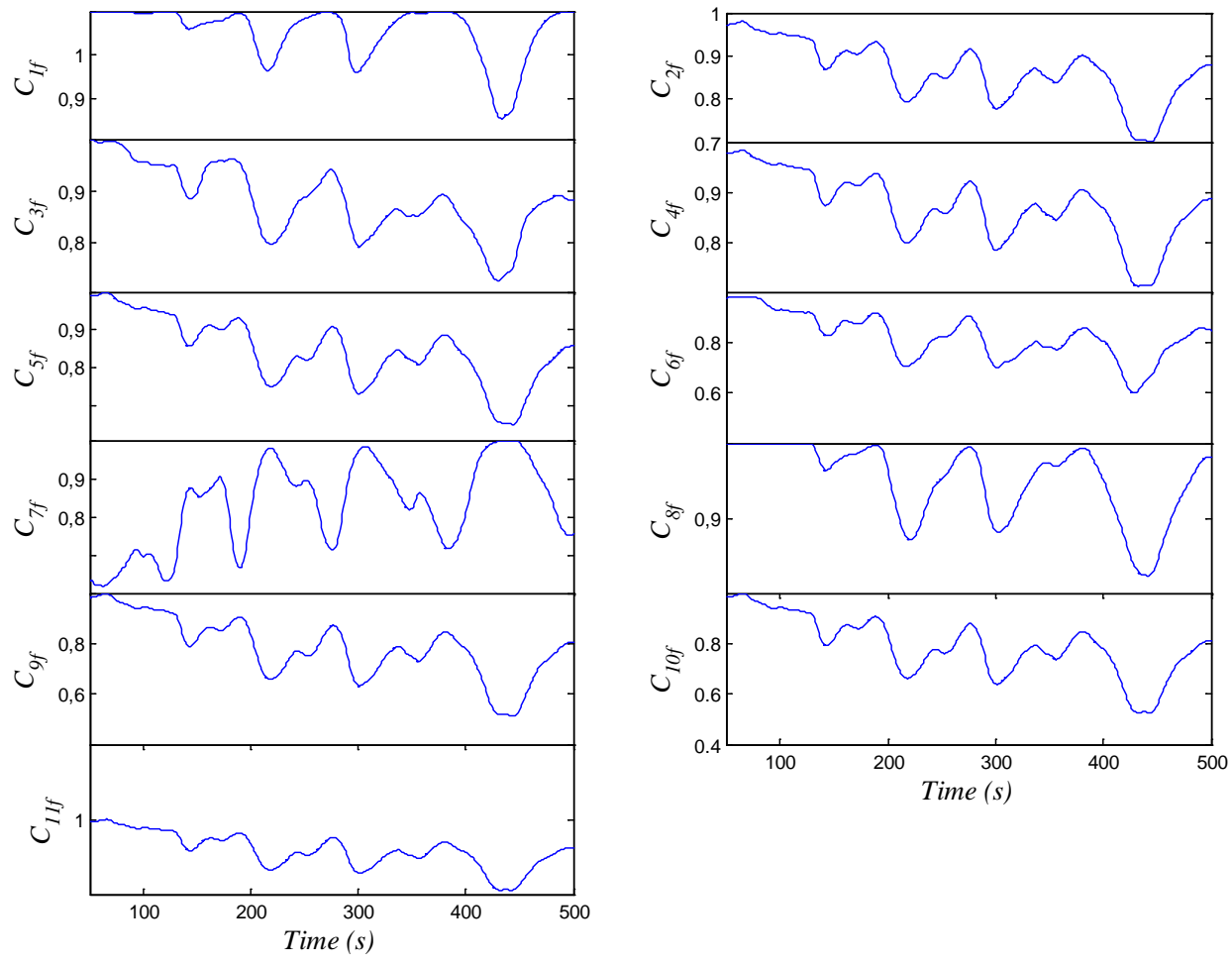

Figure 4. Filtered flame image information

\subsection{Regression Analysis with Matrix Approximation}

The use of matrices allows more compact framework because it can be possible to represent the observations, levels of repressors variables, regression coefficients, and random errors in terms of vector [24]. The matrix-approximated model is given in Eq. (2).

$$
Y=X \beta+\varepsilon
$$

Eq.(2) can be rewritten as in Eq. (3) by using of matrix notation.

$$
\left[\begin{array}{c}
y_{1} \\
y_{2} \\
\vdots \\
y_{n}
\end{array}\right]=\left[\begin{array}{cccc}
1 & x_{11} & \ldots & x_{1 k} \\
1 & x_{21} & \ldots & x_{2 k} \\
\vdots & \vdots & \ddots & \vdots \\
1 & x_{n 1} & \cdots & x_{n k}
\end{array}\right]\left[\begin{array}{c}
\beta_{1} \\
\beta_{2} \\
\vdots \\
\beta_{k+1}
\end{array}\right]+\left[\begin{array}{c}
\varepsilon_{1} \\
\varepsilon_{2} \\
\vdots \\
\varepsilon_{m}
\end{array}\right]
$$

Where $\mathrm{Y}$ is a $\mathrm{nx} 1$ dimensional vector containing the outputs of the model, $\mathrm{X}$ is a $\mathrm{nx}(\mathrm{k}+1)$ dimensional matrix containing the regression variables, $\hat{\beta}$ is the vector containing the smallest squares estimator and $\varepsilon$ is the vector containing $\mathrm{nx} 1$ dimensional errors.

The first step in matrix-assisted multiple linear regression analysis is to determine the smallest squares estimator vector $\widehat{\beta}$. $\widehat{\beta}$ is defined by the equation given in Eq.(4) [24].

Here, $\hat{\beta}$ is drawn as in Eq. (4).

$$
X^{T} X \hat{\beta}=X^{T} Y
$$

The number of rows of vector is $n=500$. On the other hand, the information set generated instantaneously from the images taken from the CCD camera is assigned as $X_{1}$ independent variable vector and also the data set which is generated with flue gas temperature data assigned as $X_{2}$ independent variable vector. Therefore, the size of the matrix $X$ is nx3. Note that the first column of the matrix $X$ consists of ones. Eleven different regression analyzes were performed with the experimental data sets constructed in the way mentioned above. In each regression analysis, different image properties are used $\left(C_{1 f}, C_{2 f}, \ldots, C_{11 f}\right)$. For instance, in the first analysis, 'one norm' of the source matrix of frame image which is obtained per second from CCD camera is assigned as $X_{1}$ independent variable vector. The results of the regression analyzes for 11 different properties of the flame images are given in Table 1 . The obtained $\lambda$ estimation correlations which are corresponds to the filtered image matrix parameters are denoted as $\lambda_{t 1}$, $\lambda_{t 2}, \ldots, \lambda_{t 11}$, respectively. According to this analysis, it can be seen that the matrix trace parameter is related to $\lambda$ with a minimum error. Norm-based image feature performances gave results which are similar to each other as expected. The predicted $\lambda$ values which are obtained by using regression relations are given in Figure 5 for each model separately. It is seen that filtered trace value of the flame image, $C_{6 f}$ can follow the air excess coefficient $\lambda$ better than the other image properties. Figure 5 shows that norm-based image properties produce close results. The similarity of the properties in the results of regression analysis also indicates the consistency of the analysis. For example, the regression results for $C_{2 f}$ and $C_{4 f}$ performances that seems very close to each other in Figure 5 are $13.53 \%$ and $13.58 \%$, respectively. 
Table 1. Regression analysis results

\begin{tabular}{|c|c|c|c|}
\hline Filtered variables & Symbol & Regression relation & Maximum error \\
\hline One norm & $C_{1 f}$ & $\lambda_{t 1}=2.9700-2.4510 * C_{1 f}+0.1835 * T_{\text {flue }}$ & $\% 16.74$ \\
\hline Two norm & $C_{2 f}$ & $\lambda_{t 2}=1.7963-1.0272 * C_{2 f}-0.1535 * T_{\text {flue }}$ & $\% 13.53$ \\
\hline Infinity norm & $C_{3 f}$ & $\lambda_{t 3}=1.8350-1.0177 * C_{3 f}-0.1858 * T_{\text {flue }}$ & $\% 13.62$ \\
\hline Frobenius norm & $C_{4 f}$ & $\lambda_{t 4}=1.8429-1.0573 * C_{4 \tau^{-}}-0.1668^{*} T_{\text {flue }}$ & $\% 13.58$ \\
\hline Sum of normalized pixels & $C_{5 f}$ & $\lambda_{t 5}=1.0876-0.2709 * C_{5 f}-0.1168 * T_{\text {flue }}$ & $\% 31.83$ \\
\hline Matrix trace & $C_{6 f}$ & $\lambda_{t 6}=1.6353-0.8118 * C_{6 f}-0.2171 * T_{\text {flue }}$ & $\% 11.69$ \\
\hline Matrix rank & $C_{7 f}$ & $\lambda_{t 7}=1.5122+0.4354 * C_{7 f}-1.1531 * T_{\text {flue }}$ & $\% 20.81$ \\
\hline $\begin{array}{l}\text { Sum of the pixels with a threshold Value greater } \\
\text { than } 30\end{array}$ & $C_{8 f}$ & $\lambda_{t 8}=1.3348-0.2205 * C_{8 f}-0.3930 * T_{\text {flue }}$ & $\% 24.98$ \\
\hline Smallest eigenvalue & $C_{9 f}$ & $\lambda_{t 9}=0.8519-0.3284 * C_{9 f}+0.1583 * T_{\text {flue }}$ & $\% 33.51$ \\
\hline Sum of the smallest 10 eigenvalues & $C_{10 f}$ & $\lambda_{\text {t10 }}=0.8795-0.3240 * C_{10 f}+0.1278 * T_{\text {flue }}$ & $\% 33.48$ \\
\hline Sum of all eigenvalues & $C_{11 f}$ & $\lambda_{\text {t11 }}=0.8836-0.3230 * C_{11 f}+0.1231 * T_{\text {flue }}$ & $\% 33.45$ \\
\hline
\end{tabular}

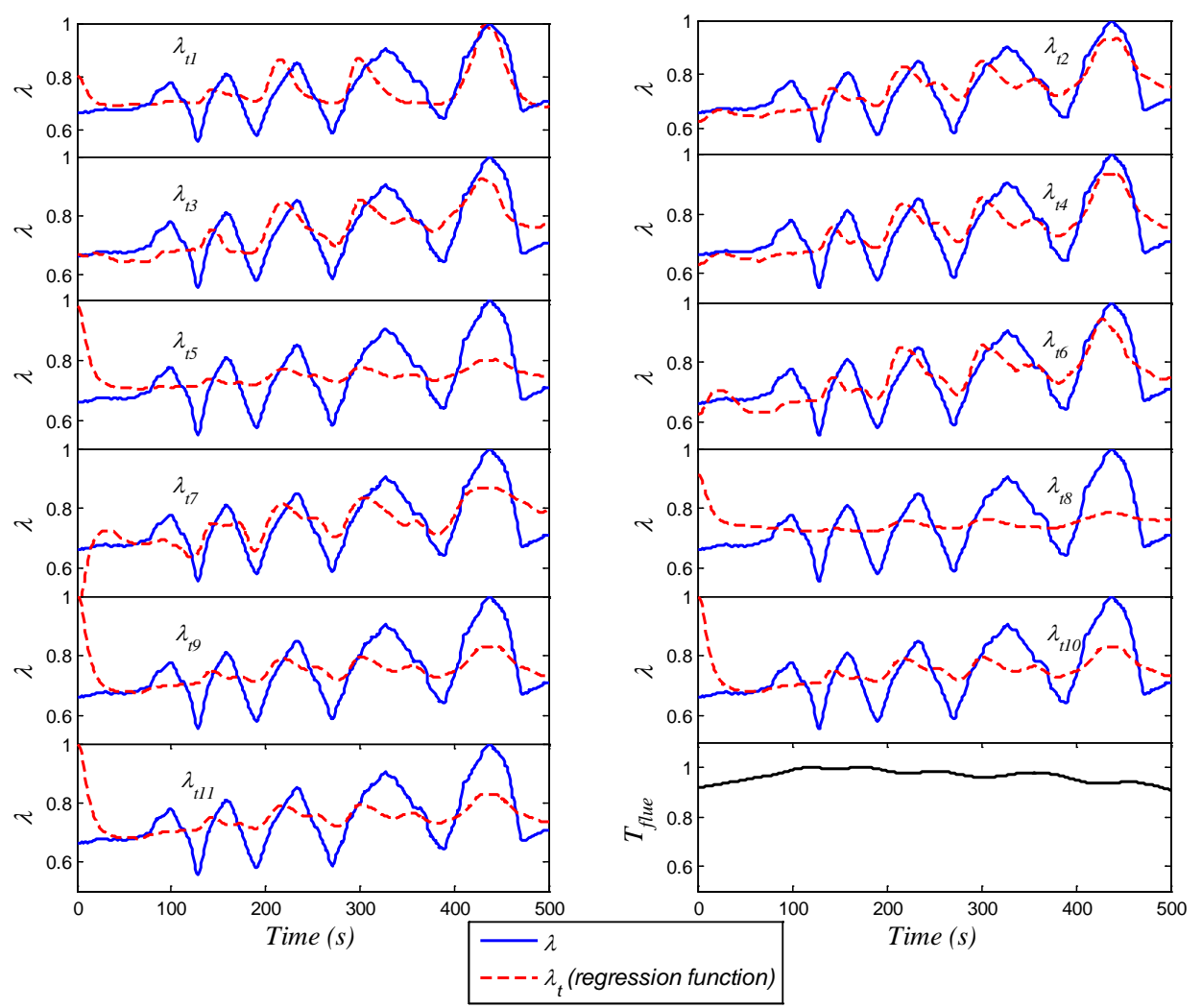

Figure 5. Estimated $\lambda$ values by regression model.

The accuracy of the regression models obtained in this way is presented comparatively. With respect to the results obtained from this regression analysis performed on 11 different flame characteristic features, it was found that the matrix trace had the most valuable correlation to the air excess coefficient.

\section{Prediction System}

The filtered trace data has been then evaluated together with flue gas temperature values in a neural network (NN).
The processing flow diagram is given in Figure 6.

The NN is trained by using of the Levenberg-Marquardt algorithm. This algorithm typically takes more memory but less time. Training automatically stops when generalization stops improving, as indicated by an increase in the mean square error of the validation samples. Only one hidden layer is used in the NN. One hundred neurons are used in the hidden layer. 70 percent of the data is used for training. Regression results are given in Figure 7. Accordingly, proposed prediction algorithm fits in with actual data of $\lambda$ in $\mathrm{R}=0.984$. 


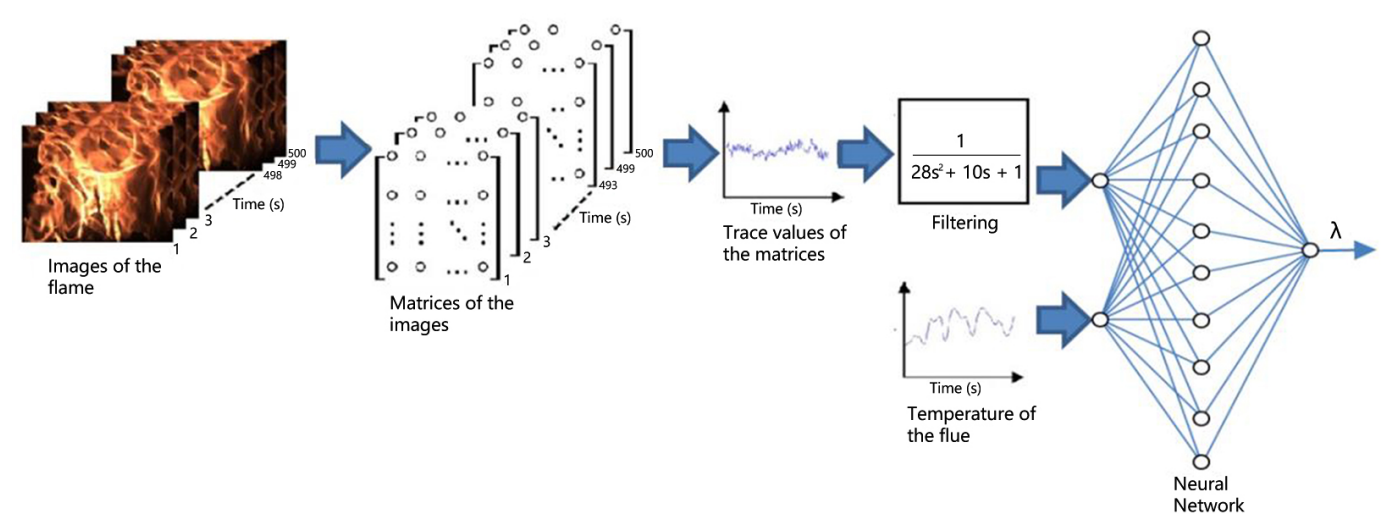

Figure 6. Flow diagram of the proposed system.
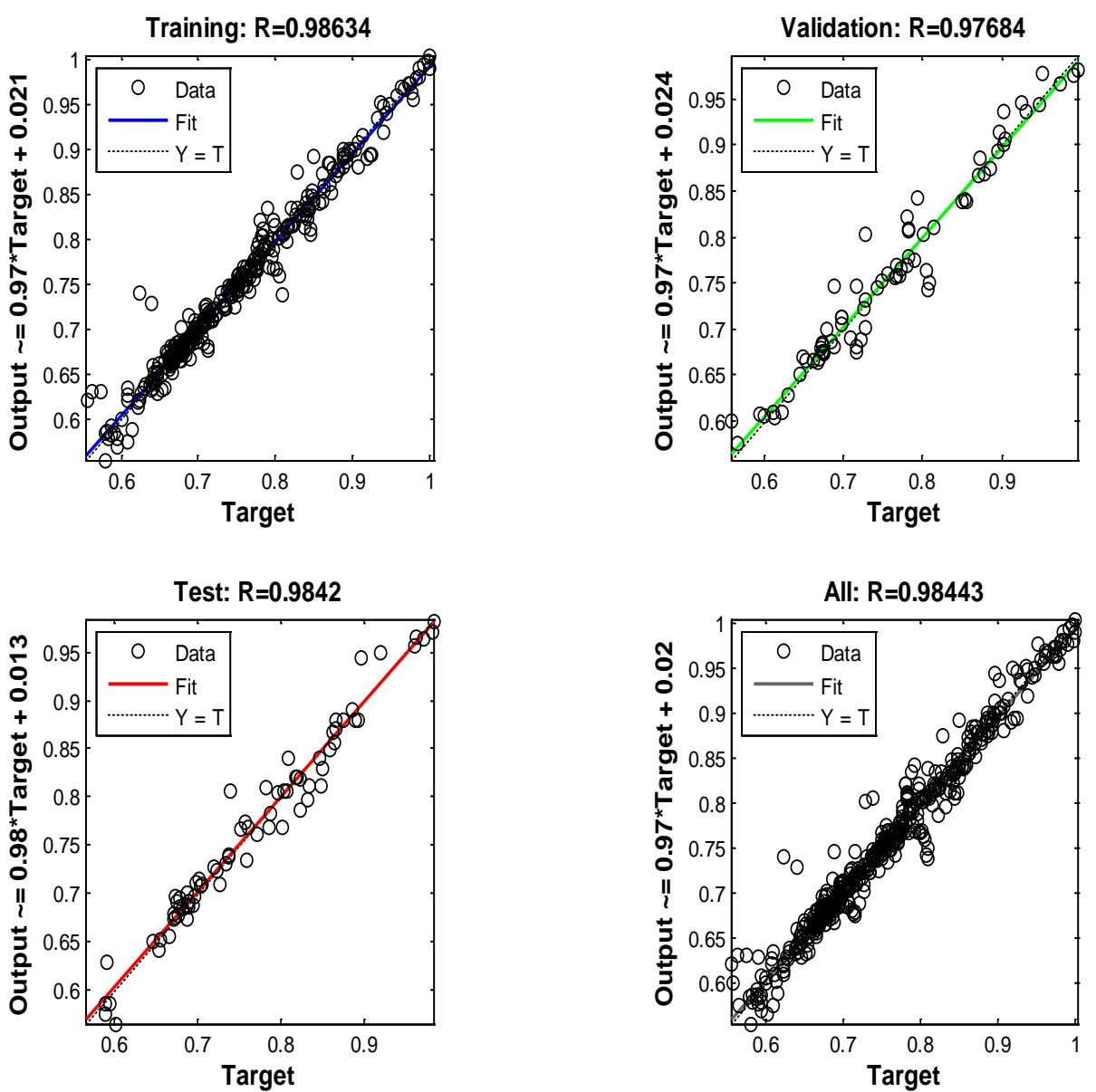

Figure 7. Regression results of the proposed system.

Prediction performance of the proposed system is given in Figure 8. First 350 data is used for training. Prediction data produced by the proposed system is noiseless. This is very important in designing of closed loop control systems.

The high accurcy ANN model then subjected to a validation experiment. For 1000 seconds, actual and predicted values of excess air coefficient were acquired from combuster. The results have showed that proposed ANN model achieved a perfect estimation performance. Validation experiment results of ANN model is given in Figure 9. 


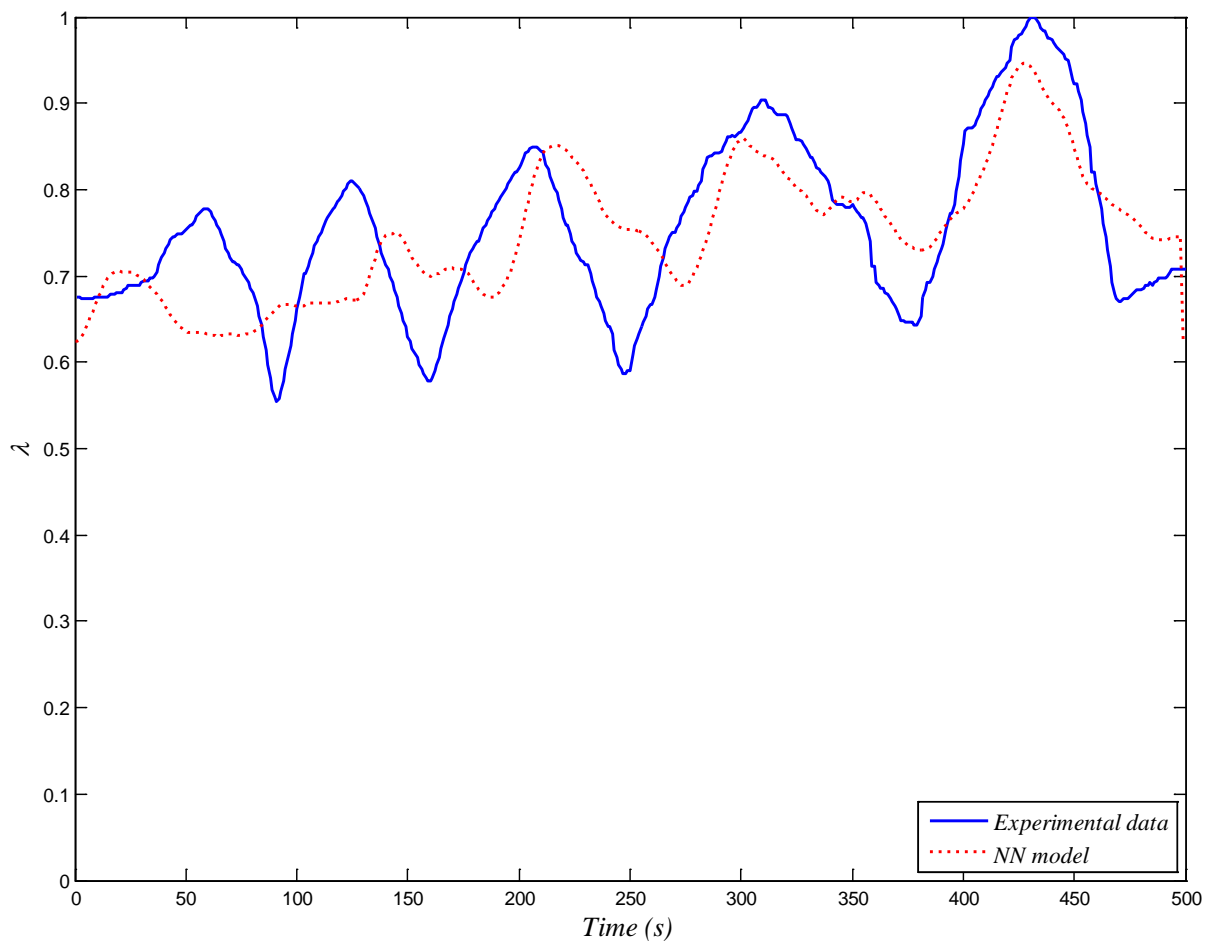

Figure 8. Performance of the proposed system.

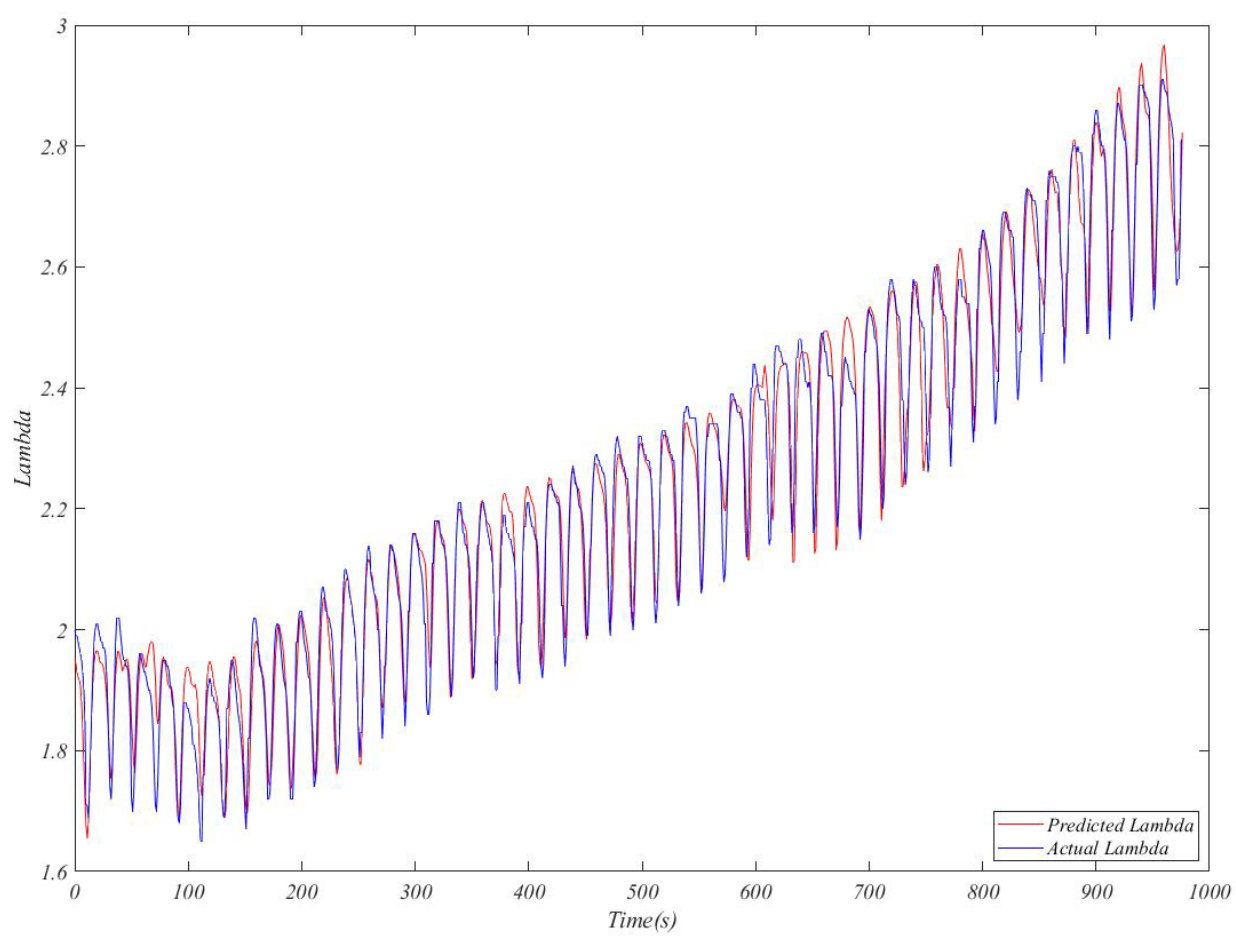

Figure 9. Validation results of the proposed system. 


\section{Conclusions}

Excess air coefficient is one of the most important parameter characterizing efficiency of the combustion. The ideal value of excess air factor offers a good reference for closed loop combustion control system. In this study, a basic prediction algorithm of excess air coefficient is proposed for automatically feeding coal burners equipped with a CCD camera. Trace of flame image matrix and temperature of flue are inputs in the prediction algorithm. In the algorithm, instantaneous trace signal is passed a low pass filter to remove high frequency noise. The proposed algorithm can estimate the excess air coefficient with high precision. In the regression analyze, it is reached $\mathrm{R}=0.984$ in terms of accuracy. Furthermore, predicted data is clean in the way of its using in the closed loop control systems. In next studies, the proposed algorithm can be used for constituting closed loop combustion control systems.

\section{Acknowledgements}

This work was supported by The Scientific and Technological Research Council of Turkey (TUBITAK, Project number: 117M121) and MIMSAN AS.

\section{REFERENCES}

[1] C. Onat, WGC based robust and gain scheduling PI controller design for condensing boilers. Advances In Mechanical Engineering, Vol.2014, pp.1-10, 2014.

[2] C. Onat, S. Canbazoğlu, An investigation on the economic analysis of wind turbines, Energy Exploration \& Exploitation, Vol: 25 (1), pp:27-37, 2007

[3] C. Onat, S. Canbazoğlu, Wind energy potential of Southeast Anatolia Region of Turkey and an optimum wind turbine propeller design for Siverek, Energy Exploration \& Exploitation, Vol: 25 (2), pp:107-121, 2007

[4] F. Li, Z. Cao, L. Xu and Y. Xie, Prediction of Equivalence Ratio in Pulse Combustor from Ion Current Amplitude Spectrum, Fuel, pp. 179-187, 2018.

[5] C. Onat, S.E. Hamamci and S. Obuz, A Practical PI Tuning Approach For Time Delay Systems. Proceedings of the 10-th IFAC Workshop on Time Delay Systems, IFAC Proceedings Volumes, Vol: 45(14), pp.102-107, 2012.

[6] C. Onat, A New Concept on PI Design For Time Delay Systems: Weighted Geometrical Center. International Journal of Innovative Computing Information And Control. Vol.9, pp.1539-1556, 2013.

[7] C. Onat, A New Design Method for PI-PD Control of Unstable Processes with Dead Time. ISA Transactions, Vol: 84, pp: 69-81, 2019.

[8] M.M. Ozyetkin, C. Onat, N. Tan, PID Tuning Method for Integrating Processes Having Time Delay and Inverse
Response, IFAC-Papers On Line, Vol: 51 (4), pp:274-279, 2018.

[9] L. Bertucco, A. Fichera, G. Nunnari and A. Pagano, A cellular neural networks approach to flame image analysis for combustion monitoring, Proc. 6th IEEE Int. Workshop Cell, Neural Networks Application. pp.455-459, 2000.

[10] G. Lu, Y. Yan and M. Colechin, A digital imaging based multifunctional flame monitoring system. IEEE Transactions on instrumentation and measurement. Vol.53, No.4, p.1152-1158, 2004.

[11] M. Shimoda, A. Sugano, T. Kimura, Y. Watanabe and K. Ishiyama, Prediction methods of un-burnt carbon for coal fired utility boiler using image processing technique of combustion flame. IEEE Trans. Energ. Convers. Vol.5, pp.640-645, 1990.

[12] Y. Yan, G. Lu and M. Colechin, Monitoring and characterization of pulverized coal flames using digital imaging techniques, Fuel. Vol.81, pp.647-656, 2002.

[13] H.C. Zhou, C. Lou, Q. Cheng and Z.F. Huang, Experimental investigations on visualization of three-dimensional temperature distributions in a large-scale pulverized-coal fired boiler furnace, Proc. Combust. Inst. Vol.30, pp.16991706, 2005

[14] M.G. Allen, An imaging neural network combustion control system for utility boiler application, Combust. Flame. Vol.94, pp.205-214, 1993.

[15] H. Zhou and C. Han, An exploratory investigation of the computer-based control of utility coal-fired boiler furnace combustion, J. Eng. Therm. Energy Power. Vol.2, p.111116, 1994.

[16] H. Zhou, Z. Luo, L. Wu and B. Huang, A new direct energy balance control concept for pc-fired boiler-turbine unit based on radiation energy signal, in: The 17th Annual Joint ISA POWID/EPRI Controls and Instrumentation Conference. Vol.471, pp. 1-12, 2007.

[17] B. Huang, Z. Luo and Zhou, Optimization of combustion based on introducing radiant energy signal in pulverized coal-fired boiler, Fuel Process. Technol. Vol.91, pp.660668, 2010.

[18] H. Zhou, Q. Tang, L. Yang, Y. Yan, G. Lu and K. Cen, Support vector machine based online coal identification through advanced flame monitoring, Fuel. Vol.117, pp.944-951, 2014.

[19] Z. Luo and H. Zhou, A combustion-monitoring system with 3-D temperature reconstruction based on flame-image processing technique, IEEE $\mathrm{T}$ Instrum. Meas. Vol.56, pp.1877-1882, 2007.

[20] Z. Luo, F. Wang, H. Zhou, R. Liu, W. Li and G. Chang, Principles of optimization of combustion by radiant energy signal and its application in a 660 MWe down and coal-fired boiler, Korean J. Chem. Eng. Vol.28, pp.23362343, 2011.

[21] Z. Guoyi, Q. Jianwei, S. Yipeng, L. Zhonggen, L. Zixue and Z. Huaichun, Experimental detection of radiative energy signal from a supercharged marine boiler and simulation on its application in control of drum water level, Appl. Therm. Eng. Vol.31, pp.3168-3175, 2011. 
[22] M.F. Talu, C. Onat and M. Daskin, Prediction of Excess Air Factor in Automatic Feed Coal Burners by processing of Flame Images, Chinese Journal of Mechanical Engineering. Vol.30, pp.1-10, 2017.

[23] X. Bai, Lu, G., Hossain, M. M., Szuhánszki, J., Daood, S. S., Nimmo, W., \& Pourkashanian, M. (2017). Multi-mode combustion process monitoring on a pulverised fuel combustion test facility based on flame imaging and random weight network techniques. Fuel, 202, 656-664.

[24] S.H. Brown, Multiple Linear Regression Analysis: A Matrix Approach with MATLAB, Alabama Journal of Mathematics, Vol.34, pp.1-3, 2009. 